\title{
The interaction between dietary Non-Enzymatic Antioxidant Capacity (NEAC) and variant of Cocaine and Amphetamine-Regulated Transcript Prepropeptide (CARTPT) 5q13-14 - rs2239670 locus on hypothalamic-pituitary axis hormones and metabolic risk factors in obese individuals
}

Mahdieh Khodarahmi

Tabriz University of Medical Sciences

Mahdieh Farhangi ( $\nabla$ abbasalizadfarhangim@gmail.com )

Tabriz University of Medical Sciences

Mahdi Niknam

Tabriz University of Medical Sciences

\section{Research}

Keywords: Obesity, CARTPT, Gene-diet interaction, polymorphism, TAC, metabolic factors

Posted Date: July 29th, 2020

DOI: https://doi.org/10.21203/rs.3.rs-50351/v1

License: (c) (i) This work is licensed under a Creative Commons Attribution 4.0 International License. Read Full License 


\section{Abstract}

\section{Background}

Epidemiological studies have illustrated that polymorphisms of Cocaine and Amphetamine-Regulated Transcript Prepropeptide gene (CARTPT) may affect obesity outcomes. However, no study has investigated the CARTPT gene-diet interactions in term of antioxidant potential of diet. This study aimed to evaluate the interactions between CARTPT gene and dietary non-enzymatic antioxidant capacity on hypothalamic- pituitary axis hormones and cardio-metabolic risk factors.

Method and Material

In the present cross-sectional study, 288 apparently healthy obese adults aged 20-50 years with a body mass index (BMI) of $30-40 \mathrm{~kg} / \mathrm{m}^{2}$ completed the study. Dietary assessment was performed by food frequency questionnaire (FFQ) and antioxidant capacity of diet was estimated by calculating the oxygen radical absorbance capacity (ORAC), ferric reducing antioxidant power (FRAP), total radical trapping antioxidant parameter (TRAP) and Trolox equivalent antioxidant capacity (TEAC). The CARTPT rs2239670 polymorphism was genotyped by polymerase chain reaction-restriction fragment length polymorphism (PCR-RFLP) technique.

Results

There was a significant interaction between CARTPT rs2239670 and dietary ORAC on BMI ( $P_{\text {interaction }}=$ 0.048 ) and fat mass percent ( $F M \%)\left(P_{\text {interaction }}=0.008\right)$; in A allele carriers, higher adherence to the dietary ORAC was related to lower level of $\mathrm{BMI}$ and $\mathrm{FM} \%$. As well as, the significant interactions were observed between FRAP index and rs2239670 in relation to HOMA $\left(P_{\text {interaction }}=0.049\right)$ and QUICKI $(P$ interaction $=0.048)$. Moreover, there were significant interactions of rs 2239670 with TRAP $\left(P_{\text {interaction }}=\right.$ 0.029) and TEAC $\left(P_{\text {interaction }}=0.034\right)$ on the serum glucose level; individuals with AG genotype were more respondent to higher intake of TRAP.

Conclusion

The present study indicated the interactions between CARTPT genotypes and dietary NEAC on obesity and its-related metabolic parameters. Further studies are warranted to confirm our findings.

\section{Background}

Obesity, as a worldwide pandemic, has become a global public health problem and its prevalence is rising at an alarming rate in many countries [1]. The worldwide prevalence of overweight and obesity has doubled since 1980 in all age-sex groups regardless of race, ethnicity and socioeconomic status [2]. Obesity is a complex and preventable disease which substantially increases risk of multiple comorbidities such as: diabetes mellitus, cardiovascular diseases (CVDs), hypertension, fatty liver disease, several types 
of cancers and stroke.. Thereby it can affect quality of life, life expectancy, work productivity and healthcare costs [1]. There is scientific evidence that obesity plays an important role in the initiation and progression of these pathological conditions through oxidative stress which is characterized by elevated reactive oxygen species (ROS) and/or reduced antioxidant capacity [3]. Oxidative stress damages or alterations in antioxidant defenses have been implicated in the pathogenesis and development of obesity-related consequences such as CVDs and cancer [3-5]. Emerging evidence suggest that the development of obesity, as a multifactorial disorder, and its-related co-morbidities is determined by interactions between genetic and environmental variables particularly diet [6]. Accumulating evidence suggests that intake of dietary antioxidants (e.g. selenium, vitamin $C$ and vitamin $E$ ) through nonenzymatic defense mechanisms can protect against cell damage caused by oxidative stress and also related inflammatory outcome [7]. In this regard, the epidemiologic studies have reported an inverse association between intakes of fruits and vegetables, which are rich source of antioxidants, and the risk of several chronic conditions such as CVDs and cancers [8,9]. Since various antioxidants are combined in foods and they act in a cumulative and synergistic way in complex matrixes, assessment of single specific antioxidant may not reflect the potential overall antioxidant effect of the diet. Thus, investigation of the dietary total antioxidant capacity (TAC) can provide a better tool to examine the beneficial effects of dietary antioxidants on chronic diseases prevention [10]. Non-enzymatic antioxidant capacity (NEAC), also recognized as TAC, is a new approach to capture synergistic effects of antioxidants in diet and generally can be estimated through four different methods including: Trolox equivalent antioxidant capacity (TEAC), ferric reducing-antioxidant power (FRAP), total radical-trapping antioxidant parameter (TRAP) and oxygen radical absorbance capacity (ORAC) [11]. Recently, observational studies have shown that high dietary NEAC is inversely related to the risk of stroke, diabetes and different cancers [12-14].

Since obesity is highly heritable, genetic factors substantially contribute to development of obesity and its serious consequences [15]. Cocaine- and amphetamine-regulated transcript prepropeptide gene (CARTPT), which is located at the chromosome 5q13-14 and expresses the cocaine- and amphetamineregulated transcript (CART) protein, has been recognized to be a susceptibility locus for obesity (Fig. 1). CART protein, as one of the various neuropeptides in the arcuate nucleus (ARC) of the hypothalamus, is involved in the regulation of food intake and body weight [16]. In this regard, previous evidence have shown that, CART peptides which are co-expressed with other neurotransmitters such as a-melanocytestimulating-hormone (a-MSH) inhibit food intake [17]. Recent studies have indicated that polymorphisms in the CARTPT gene might influence obesity, metabolic syndromes (MetS) and its components [18]. However, the results of studies regarding these associations are controversial [19]. These heterogeneous associations may be due to the complex pathogenesis and etiology of obesity which involve interaction between genetic and environmental factors especially diet. A positive association between CARTPT rs2239670 variant and alcoholism has been reported in the Korean population and as obesity [20] and substance abuse have a common neurobiological basis, investigation of the association between this polymorphism and obesity and metabolic factors is interested.

Therefore, since the better understanding of the gene-diet interactions can provide more efficient strategies for personalized treatment, and also to our knowledge, no studies have assessed the 
modification effects of diet in term of dietary antioxidant capacity on the association of CARTPT gene, we aimed to with cardio-metabolic risk factors and hypothalamic hormones, we aimed to evaluate the potential interactions between CARTPT rs2239670 and dietary NEAC on cardio-metabolic risk factors and hypothalamic hormones among obese population.

\section{Method And Material: \\ 2.1. Study participants}

This cross-sectional study was conducted in Tabriz, the city in the north-west of Iran, from December 2017 to April 2019 among apparently healthy obese subjects using convenience sampling method. Study participants included 288 adult subjects (20-50 years old) who were obese (body mass index (BMI) $\geq$ $30 \mathrm{~kg} / \mathrm{m}^{2}$ ). Detailed illustration of recruitment of research participants has been provided elsewhere [21]. Individuals were excluded if they met any of the following criteria: menopause, pregnancy, lactation, a history of cardiovascular diseases, hypertension, hyperlipidemia, cancer, type 2 diabetes mellitus, renal diseases, taking any medication affecting the variables studied (hypoglycemic, lipid-lowering, antihypertensive, corticosteroids, or antidepressants). The written informed consent was obtained from all the participants at the beginning of the study. The study protocol was approved and registered by the ethical committee of Tabriz University of Medical Sciences (Ethics number: IR.TBZMED.REC.1397.266).

\subsection{Demographic and anthropometric assessments}

Information of physical activity and other demographic characteristics (age, sex) were collected by trained interviewer at the beginning of the study. Physical activity level was also estimated using the short form of the international physical activity questionnaire (IPAQ) [22]. Anthropometric measurements including weight, height, waist circumference (WC), and hip circumference (HC) were conducted by a trained dietitian. Weight was measured in minimally clothed subjects using a Seca scale (Seca, Germany) to the nearest $0.01 \mathrm{~kg}$. Height was measured in light clothing and without shoes by means of a tape measure with a precision of $0.1 \mathrm{~cm}$. The narrowest level and the largest part were used to measure the waist and hip circumference (WC and $\mathrm{HC}$ ), respectively, with an unscratched tape without applying any pressure to the body. Assessments of body composition were performed using bioelectrical impedance analysis (BIA) technology (Tanita, BC-418 MA, Tokyo, Japan). Waist-to-hip ratio (WHR) was calculated by dividing WC to HC. Systolic and diastolic blood pressure (SBP and DBP) were measured after at least 10 min of rest in the seated position. This measurement was carried out two times and the average of them was considered as subjects BP.

\subsection{Biochemical measurements}

Blood samples were obtained from each study participant after 10-12 hour of fasting. Plasma and serum were prepared by centrifugation $\left(10 \mathrm{~min}\right.$ at $4500 \mathrm{rpm}, 4^{\circ} \mathrm{C}$ ) and their aliquots were stored at $80^{\circ} \mathrm{C}$ until assay. Serum concentrations of glucose, total cholesterol (TC), triglyceride (TG) and highdensity lipoprotein cholesterol (HDL-C) were measured by commercial kits (Pars Azmoon Inc, Tehran, 
Iran). As well as, serum low-density lipoprotein cholesterol level (LDL-C) was calculated using friedewald equation. Insulin, a-MSH and AgRP concentrations were analyzed by enzyme-linked immunosorbent assay kits (Bioassay Technology Laboratory, Shanghai Korean Biotech, Shanghai City, China) based on manufacturer's instructions. Moreover, following formulas were applied to compute homeostasis model assessment insulin resistance index (HOMA-IR) and quantitative insulin sensitivity check index (QUICKI) values.

HOMA-UR $=[$ fasting glucose $(\mathrm{mmol} / \mathrm{L}) \times$ fasting insulin $(\mu \mathrm{lU} / \mathrm{mL})] / 22.5[23]$

QUICKI = 1 / [log fasting glucose $(\mathrm{mmol} / \mathrm{L})+$ log fasting insulin $(\mu \mathrm{lU} / \mathrm{mL})][24]$

\subsection{Dietary assessments and dietary TAC calculation}

Dietary intake of participants over the last year was evaluated using a 147-item semi-quantitative food frequency questionnaire (FFQ) which was validated in the previous studies $[25,26]$. Interviewer asked participants to report their frequency and amount of each food item during the previous year based on a daily, weekly, monthly and yearly basis. Portion sizes of recorded food items were converted to grams by using household measures [27].Then, daily nutrient consumption was analyzed by Iranian Food Composition Table (FCT) [28]. Information missing from this FCT was completed with the United States Department of Agriculture FCT [29].

NEAC is an indicator of diet quality which describes the ability of food antioxidants to scavenge free radicals. To estimate the dietary NEAC for each subject, four assays were used: ferric reducingantioxidant power (FRAP) [30] which measures the ability of dietary antioxidants to reduce ferric to ferrous ions, total radical-trapping antioxidant parameter (TRAP) [31] that evaluates the chain-breaking antioxidant potential for scavenging peroxyl radicals, oxygen radical absorbance capacity (ORAC) [32] which measures the antioxidant capacity against peroxyl radicals using an area-under-curve (AUC) technique and Trolox equivalent antioxidant capacity (TEAC) [31] which is based on scavenging ability of antioxidants against a radical cation in both lipophilic and hydrophilic environments, Since maillard products of the coffee, which is produced during roasting process, are the main contributors to the high antioxidant capacity of coffee in vitro [33] and also its still unknown whether these products are efficiently absorbed and exert an antioxidant effect in vivo [34], we decided to exclude the contribution of coffee to NEAC. Dietary NEAC values for $64,63,59$ and 65 food items in the FFQ were assigned by ORAC, FRAP TRAP and TEAC, respectively. Finally, total dietary NEAC was calculated by multiplying the NEAC values of individual foods by the amount of each food consumed and then summing up these to obtain dietary NEACE for each participant.

\subsection{DNA extraction and genotyping:}

The extraction of genomic DNA from blood samples was performed by phenol/chloroform extraction method. Nano Drop 2000C spectrophotometer was used to determine the concentration and purity of the DNA extracted from each sample. Genotyping the CARTPT rs2239670 variant was carried out by polymerase chain reaction-restriction fragment length polymorphism (PCR-RFLP) method using the 
following primers: forward 5'-CCTGCTGCTGATGCTACCTCT-3' and reverse 5'GCGCTTCGATCTGCAACACAC-3'. The cycling conditions in DNA thermocycler were as follows: an initial denaturation at $94^{\circ} \mathrm{C}$ for $5 \mathrm{~min}, 35$ cycles of denaturation at $94^{\circ} \mathrm{C}$ for $30 \mathrm{~s}$, annealing at $60{ }^{\circ} \mathrm{C}$ for $30 \mathrm{~s}$, extension at $72{ }^{\circ} \mathrm{C}$ for $20 \mathrm{~s}$, and final extension at $72{ }^{\circ} \mathrm{C}$ for $10 \mathrm{~min}$. PCR amplification was carried out in a final volume of $25 \mu \mathrm{l}$ which included $2 \mu \mathrm{l}$ genomic DNA, $12.5 \mu \mathrm{l}$ distilled water, $10 \mu \mathrm{l}$ Taq DNA Polymerase Master Mix (Ampliqon; Denmark) and $0.5 \mu$ of each primer. PCR product was digested with

Apal restriction enzyme (Takara, Japan) and then digested product was subjected to electrophoresis on $3 \%$ agarose gel. Fragments containing three possible genotypes of the CARTPT rs2239670 were detected: uncut homozygous AA (552 bp), cut heterozygous AG (212, 340 and 552 bp) and cut homozygous GG (340 and $212 \mathrm{bp}$ ). To confirm the results of PCR-RFLP, 10 percent of PCR samples were directly sequenced.

\subsection{Statistical analyses}

Normal distribution of data was checked by Kolmogorov-Smirnov test. The comparison of qualitative and quantitative variables was conducted by chi- square test and analysis of variance (ANOVA), respectively. Data on continuous and categorical variables were presented as the mean $\pm S D$ and the frequencies or percentages, respectively. ANCOVA multivariate interaction model was used to estimate the interactions of CARTPT rs2239670 polymorphism with dietary NEAC in relation to cardio-metabolic risk factors and hypothalamic hormones after adjusting for confounding factors. All statistical analyses were performed using the Statistical Package for Social Sciences (SPSS, Chicago, II, USA) version 22.0. A p. value $<0.05$ was considered as statistically significant.

\section{Results}

The present cross-sectional study was conducted on 288 individuals ( $51.1 \%$ men and $48.9 \%$ women). The mean (SD) age and BMI of the participants were 38.04 (7.47) years and $34.72(3.88) \mathrm{kg} / \mathrm{m}^{2}$, respectively. General characteristics of participants according to CARTPT rs2239670 genotypes have shown in Table 1. The genotype and allele frequencies for the CARTPT rs2239670 polymorphism among different tertiles of the dietary NEAC are shown in Table 2. The total frequencies of genotypes among population studied were as follow: AA (10.76\%), AG (20.13\%) and GG (69.9\%). As well as, the minor frequency allele observed in this study was $20.79 \%$. No significant differences were found in the mean values of biochemical parameters of subjects across different genotypes of CARTPT rs2239670 polymorphism (Table 3). General characteristics and metabolic parameters of participants across tertiles of dietary NEAC (ORAC, FRAP, TRAP and TEAC) are provided in Table 4. There were significant differences regarding WC, BMR, TG, LDL-C and glucose across tertile categories of dietary NEAC. Although the participants in the highest tertile of TEAC had higher means of WC $(p=0.031)$ and TG $(p=0.037)$, mean of serum LDL-C level was lowest in this category $(p=0.053)$. Moreover, those in the highest tertile of the NEAC (ORAC, FRAP, TRAP, and TEAC) had higher level of BMR $(p<0.05)$. Conversely, higher means of 
glucose level $(p=0.018)$ and WC $(p=0.04)$ were seen among those in third tertile of the ORAC and FRAP, respectively.

Table 1

General characteristics of participants according to CARTPT rs2239670 polymorphism

\begin{tabular}{|c|c|c|c|c|}
\hline \multirow[t]{2}{*}{ Variables } & \multicolumn{3}{|l|}{ Genotype } & \multirow[t]{2}{*}{$P^{*}$} \\
\hline & AA & AG & GG & \\
\hline \multicolumn{5}{|l|}{ Gender } \\
\hline Male & $8(5.4)$ & $30(20.4)$ & $109(74.1)$ & \multirow[t]{2}{*}{$0.057^{a}$} \\
\hline Female & $22(15.6)$ & $28(20)$ & $90(64.4)$ & \\
\hline Age (year) & $37.05(7.45)$ & 39.78 (8.33) & $37.75(7.10)$ & 0.288 \\
\hline BMI $\left(\mathrm{kg} / \mathrm{m}^{2}\right)$ & $34.35(3.83)$ & 34.99 (4.61) & $34.76(3.75)$ & 0.848 \\
\hline WC (cm) & $105.26(10.01)$ & $107.82(12.86)$ & 109.44 (8.95) & 0.204 \\
\hline WHR & $0.89(0.08)$ & $0.92(0.07)$ & $0.93(0.07)$ & 0.025 \\
\hline FM (\%) & $34.96(8.58)$ & $34.13(8.62)$ & $33.81(9.47)$ & 0.875 \\
\hline BMR (kcal) & $1749.11(312.20)$ & 1837.59 (367.53) & $1931.75(416.11)$ & 0.116 \\
\hline PA (min/week) & $1396.32(1587.27)$ & $1232.33(1677.67)$ & 2341.14 (3493.41) & 0.106 \\
\hline $\mathrm{SBP}(\mathrm{mmHg})$ & $111.58(16.51)$ & 117.28 (14.30) & 115.90 (13.88) & 0.360 \\
\hline $\mathrm{DBP}(\mathrm{mmHg})$ & $72.95(12.48)$ & 78.89 (10.42) & 76.25 (10.90) & 0.157 \\
\hline \multicolumn{5}{|c|}{$\begin{array}{l}\text { BMI, Body Mass Index; WC, Waist Circumference; WHR, Waist-to-Hip Ratio; FM, Fat Mass; BMR, Basal } \\
\text { Metabolic Rate; PA, Physical Activity; SBP, Systolic Blood Pressure; DBP, Diastolic Blood Pressure; } \\
\text { values for gender is in number of subjects (percentage) and for all other variables are presented } \\
\text { based on mead (SD). }\end{array}$} \\
\hline
\end{tabular}


Table 2

Allele frequency and overall genotype prevalence for CARTPT rs2239670 polymorphism Genotype prevalence

Allele frequency

$\begin{array}{lllll}A A & \text { AG } & \text { G } & \text { A } & \text { G }\end{array}$

ORAC

Tertile $1 \quad 10 \%(n=10) \quad 18.3 \%(n=18) \quad 71.7 \%(n=69) \quad 6.47 \% \quad 27.25 \%$

Tertile $2 \quad 11.7 \%(n=11) \quad 23.3 \%(n=23) \quad 65 \%(n=63) \quad 7.86 \% \quad 25.84 \%$

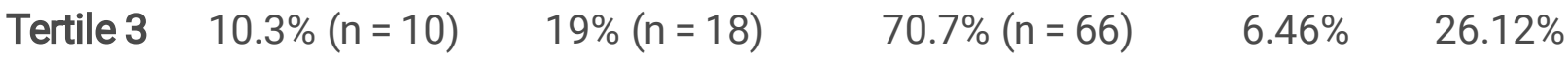

FRAP

Tertile $1 \quad 8.1 \%(n=8) \quad 22.6 \%(n=23) \quad 69.4 \%(n=69) \quad 6.72 \% \quad 28.09 \%$

Tertile $2 \quad 8.8 \%(n=8) \quad 15.8 \%(n=14) \quad 75.4 \%(n=70) \quad 5.36 \% \quad 26.68 \%$

Tertile $3 \quad 15.3 \%(n=14) \quad 22 \%(n=21) \quad 62.7 \%(n=60) \quad 8.71 \% \quad 24.44 \%$

TRAP

Tertile $1 \quad 8.2 \%(n=8) \quad 24.6 \%(n=24) \quad 67.2 \%(n=67) \quad 7.02 \% \quad 27.25 \%$

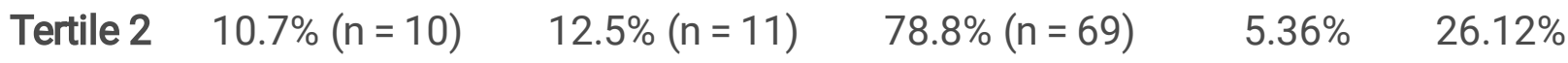

Tertile $3 \quad 13.1 \%(n=13) \quad 23 \%(n=23) \quad 63.9 \%(n=63) \quad 8.41 \% \quad 25.84 \%$

TEAC

Tertile $1 \quad 8.3 \%(n=8) \quad 25 \%(n=24) \quad 66.7 \%(n=65) \quad 7.02 \% \quad 26.68 \%$

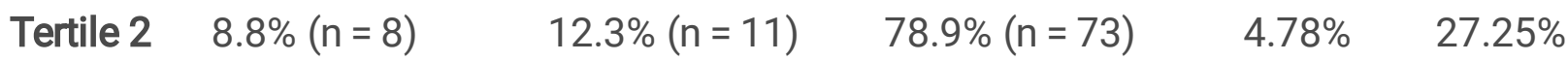

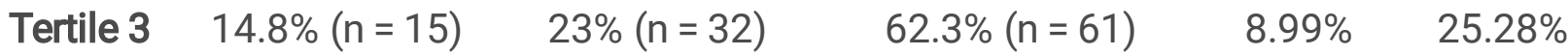

Total $\quad 10.76 \%(n=31) \quad 20.13 \%(n=58) \quad 69.09 \%(n=199) \quad 20.79 \% \quad 79.21 \%$ 
Table 3

Biochemical parameters of participants according to CARTPT rs2239670 polymorphism

\begin{tabular}{|c|c|c|c|c|}
\hline \multirow[t]{2}{*}{ Variables } & \multicolumn{3}{|l|}{ Genotype } & \multirow{2}{*}{$\mathbf{P}^{*}$} \\
\hline & AA & AG & GG & \\
\hline $\mathrm{TC}(\mathrm{mg} / \mathrm{dL})$ & 192.95 (32.75) & $185.94(32.65)$ & $188.37(34.48)$ & 0.768 \\
\hline TG (mg/dL) & $115.26(45.90)$ & $109.50(54.61)$ & $122.34(59.70)$ & 0.478 \\
\hline HDL-C (mg/dL) & $46.32(9.49)$ & $44.64(9.12)$ & $44.58(8.25)$ & 0.709 \\
\hline LDL-C (mg/dL) & $123.57(27.51)$ & 119.40 (31.98) & $119.32(31.60)$ & 0.856 \\
\hline Glucose (mg/dL) & 99.95 (22.69) & $92.67(20.11)$ & $92.53(11.76)$ & 0.137 \\
\hline Insulin ( $\mu \mid \mathrm{lU} / \mathrm{mL})$ & $17.16(10.57)$ & $12.81(6.74)$ & $16.14(8.61)$ & 0.085 \\
\hline HOMA-IR & $4.16(2.62)$ & $3.02(1.84)$ & 3.69 (2.02) & 0.109 \\
\hline QUICKI & $0.32(0.03)$ & $0.33(0.03)$ & $0.32(0.02)$ & 0.110 \\
\hline $\mathrm{a}-\mathrm{MSH}(\mathrm{ng} / \mathrm{mL})$ & $2.32(0.31)$ & $2.29(0.24)$ & $2.23(0.20)$ & 0.137 \\
\hline $\operatorname{AgRP}(\mathrm{pg} / \mathrm{mL})$ & $1.45(0.27)$ & $1.46(0.19)$ & $1.42(0.18)$ & 0.448 \\
\hline \multicolumn{5}{|c|}{$\begin{array}{l}\text { TC, Total Cholesterol; TG, Triglyceride; HDL-C, High Density Lipoprotein Cholesterol; LDL-C, Low } \\
\text { Density Lipoprotein Cholesterol; HOMA-IR, Homeostatic Model Assessment for Insulin Resistance; } \\
\text { QUICKI, Quantitative Insulin sensitivity Check Index; a-MSH, alpha-Melanocyte-Stimulating Hormone; } \\
\text { AgRP, Agouti-Related Peptide; all variables data are presented based on mean (SD). } \\
\text { * P values hased on One-Wav ANOVA }\end{array}$} \\
\hline
\end{tabular}


Table 4

General characteristics and metabolic parameters of participants across tertiles of dietary NEAC.

Tertile 1

Age (year)

ORAC

FRAP

TRAP

TEAC

WC $(\mathrm{cm})$

ORAC

FRAP

TRAP

TEAC

FM (\%)

ORAC

FRAP

TRAP

TEAC

BMR (kcal)

ORAC

1801.08 (329.37)

1780.92 (345.87)

1773.71 (336.63)

1782.41 (347.19)

TEAC

PA (min/week)

ORAC

FRAP

TRAP

TEAC

$\mathrm{SBP}(\mathrm{mmHg})$
Tertile 2

Tertile 3
$P$

0.252

0.248

0.597

0.293

$39.13(7.13)$

0.548

0.040

0.084

0.031

107.76 (10.57)

111.50 (9.54)

0.126

0.244

$33.98(9.13)$

32.34 (8.81)

0.359

33.49 (8.91)

32.82 (9.09)

0.187

$32.43(8.61)$

$33.63(9.18)$

1965.95 (361.25)

1948.65 (472.04)

0.037

1941.42 (356.25)

1994.05 (454.69)

0.007

1961.78 (482.33)

1980.71 (322.61)

0.005

1910.27 (351.02)

2022.14 (453.44)

0.003 


\begin{tabular}{|c|c|c|c|c|}
\hline & Tertile 1 & Tertile 2 & Tertile 3 & $\mathbf{P}$ \\
\hline ORAC & $116.27(14.32)$ & $117.43(13.54)$ & $114.68(15.91)$ & 0.574 \\
\hline FRAP & $113.37(11.98)$ & $118.30(17.11)$ & $116.74(13.97)$ & 0.152 \\
\hline TRAP & $115.17(12.48)$ & $115.83(15.99)$ & $117.42(15.92)$ & 0.678 \\
\hline TEAC & 114.65 (12.07) & $115.73(16.13)$ & $118.03(15.21)$ & 0.420 \\
\hline \multicolumn{5}{|c|}{$\mathrm{DBP}(\mathrm{mmHg})$} \\
\hline ORAC & $77.10(10.45)$ & $76.97(11.46)$ & $76.11(12.08)$ & 0.871 \\
\hline FRAP & $74.97(10.70)$ & $77.11(11.32)$ & $78.13(11.81)$ & 0.280 \\
\hline TRAP & $76.02(11.15)$ & $75.86(10.84)$ & 78.34 (11.89) & 0.392 \\
\hline TEAC & $76.24(11.22)$ & 75.61 (10.69) & 78.37 (11.95) & 0.360 \\
\hline \multicolumn{5}{|c|}{$\mathrm{TC}(\mathrm{mg} / \mathrm{dL})$} \\
\hline ORAC & $189.97(34.22)$ & $190.62(32.19)$ & $184.13(34.84)$ & 0.499 \\
\hline FRAP & $187.14(33.58)$ & $191.67(36.56)$ & $185.94(30.98)$ & 0.607 \\
\hline TRAP & 193.78 (36.78) & $184.95(34.28)$ & $186.02(29.43)$ & 0.278 \\
\hline TEAC & 194.08 (35.08) & $182.66(33.93)$ & $188.23(31.57)$ & 0.164 \\
\hline \multicolumn{5}{|c|}{$\mathrm{TG}(\mathrm{mg} / \mathrm{dL})$} \\
\hline ORAC & $114.81(51.75)$ & $116.79(65.47)$ & $128.27(56.90)$ & 0.382 \\
\hline FRAP & $106.67(48.15)$ & $123.94(55.37)$ & $129.29(68.29)$ & 0.075 \\
\hline TRAP & $107.01(47.46)$ & $125.06(58.86)$ & $127.81(65.96)$ & 0.094 \\
\hline TEAC & $106.40(42.35)$ & $120.16(60.34)$ & $133.18(67.12)$ & 0.037 \\
\hline \multicolumn{5}{|c|}{$\mathrm{HDL}-\mathrm{C}(\mathrm{mg} / \mathrm{dL})$} \\
\hline ORAC & $45.29(9.10)$ & $46.19(9.66)$ & $43.37(7.45)$ & 0.191 \\
\hline FRAP & $45.44(9.17)$ & $45.97(9.71)$ & $43.44(7.36)$ & 0.241 \\
\hline TRAP & $46.41(9.61)$ & $44.76(8.81)$ & $43.68(7.89)$ & 0.219 \\
\hline TEAC & $46.32(9.45)$ & $45.39(9.21)$ & $43.15(7.51)$ & 0.119 \\
\hline \multicolumn{5}{|c|}{ LDL-C (mg/dL) } \\
\hline ORAC & $121.72(30.52)$ & $121.07(28.75)$ & 115.10 (33.33) & 0.422 \\
\hline FRAP & $120.36(31.40)$ & 120.91 (33.79) & $116.64(27.41)$ & 0.705 \\
\hline TRAP & $125.96(33.09)$ & $115.18(30.82)$ & $116.78(27.88)$ & 0.107 \\
\hline
\end{tabular}




\begin{tabular}{|c|c|c|c|c|}
\hline & Tertile 1 & Tertile 2 & Tertile 3 & $\mathbf{P}$ \\
\hline TEAC & $126.47(32.62)$ & $113.23(29.87)$ & $118.44(29.11)$ & 0.053 \\
\hline \multicolumn{5}{|c|}{ Glucose (mg/dL) } \\
\hline ORAC & $91.32(10.95)$ & $92.11(13.31)$ & $101.52(34.46)$ & 0.018 \\
\hline FRAP & $91.70(11.37)$ & $98.32(33.54)$ & $94.82(16.11)$ & 0.258 \\
\hline TRAP & 91.57 (9.76) & $98.56(33.46)$ & $94.71(17.22)$ & 0.220 \\
\hline TEAC & $91.10(20.29)$ & $97.80(33.01)$ & 95.85 (17.34) & 0.232 \\
\hline \multicolumn{5}{|c|}{ Insulin ( $(\mu \mathrm{lU} / \mathrm{mL})$} \\
\hline ORAC & $16.47(10.05)$ & 14.51 (8.59) & 16.58 (8.91) & 0.365 \\
\hline FRAP & $17.11(10.52)$ & $15.62(8.54)$ & 14.81 (8.39) & 0.371 \\
\hline TRAP & $16.05(10.15)$ & 16.48 (8.98) & $15.01(8.48)$ & 0.655 \\
\hline TEAC & $16.10(10.20)$ & $16.44(8.80)$ & 14.99 (8.63) & 0.658 \\
\hline \multicolumn{5}{|c|}{ HOMA-IR } \\
\hline ORAC & 3.80 (2.59) & $3.31(2.07)$ & 4.17 (2.78) & 0.161 \\
\hline FRAP & 3.94 (2.67) & $3.82(2.69)$ & $3.51(2.14)$ & 0.623 \\
\hline TRAP & 3.71 (2.61) & $4.01(2.72)$ & $3.57(2.21)$ & 0.612 \\
\hline TEAC & $3.68(2.60)$ & $3.99(2.64)$ & $3.60(2.28)$ & 0.659 \\
\hline \multicolumn{5}{|c|}{ QUICKI } \\
\hline ORAC & $0.32(0.03)$ & $0.33(0.03)$ & $0.32(0.02)$ & 0.111 \\
\hline FRAP & $0.32(0.03)$ & $0.32(0.02)$ & $0.32(0.02)$ & 0.708 \\
\hline TRAP & $0.32(0.03)$ & $0.32(0.02)$ & $0.32(0.03)$ & 0.408 \\
\hline TEAC & $0.32(0.03)$ & $0.32(0.02)$ & $0.32(0.02)$ & 0.465 \\
\hline \multicolumn{5}{|c|}{$\begin{array}{l}\text { WC, Waist Circumference; FM, Fat Mass; BMR, Basal Metabolic Rate; PA, Physical Activity; SBP, } \\
\text { Systolic Blood Pressure; DBP, Diastolic Blood Pressure; TC, Total Cholesterol; TG, Triglyceride; HDL-C, } \\
\text { High Density Lipoprotein Cholesterol; LDL-C, Low Density Lipoprotein Cholesterol; HOMA-IR, } \\
\text { Homeostatic Model Assessment for Insulin Resistance; QUICKI, Quantitative Insulin sensitivity Check } \\
\text { Index; all variables data are presented based on mean (SD). P value based on One-Way ANOVA; follow } \\
\text { up by Tukey's post-hoc test. }\end{array}$} \\
\hline
\end{tabular}

As elucidated in Fig. 2, after adjusting for sex and age, there was a significant interaction between CARTPT rs2239670 variant and dietary ORAC on BMI $\left(P_{\text {interaction }}=0.048\right)$ and fat mass percent $(F M \%)(P$ interaction $=0.008$ ); in A allele carriers, higher adherence to the dietary ORAC was related to lower level of $\mathrm{BMI}$ and FM\%. Moreover, the significant interactions were observed between FRAP indicator and CARTPT 
rs2239670 polymorphism in relation to HOMA ( $P$ interaction $=0.049)$ and QUICKI ( $P$ interaction $=0.048)$. Such that, adherence to the dietary FRAP modified positively the association of the CARTPT rs2239670 variant with HOMA and QUICKI in G allele carriers. It seemed that dietary NEAC couldn't modulate detrimental effects of CARTPT rs2239670 polymorphism in participants carrying AA genotype. Additionally, the significant interactions were observed for CARTPT rs2239670 variant with FRAP (0.029) and TEAC (0.034) in relation to serum glucose level. The highest serum glucose concentration was observed in the AA genotype with moderate compliance with FRAP and TEAC; while high compliance with these indices couldn't effect on genetic predisposition to blood sugar abnormalities.

\section{Discussion}

To the best of our knowledge, the current research is the first attempt to study the interaction between CARTPT rs2239670 polymorphism and dietary NEAC on cardio-metabolic risk factors and hypothalamic hormones. Our finding suggest that the rs2239670 on chromosome 5q13-14 interacts with dietary ORAC, FRAP and TEAC to influence obesity and obesity-related metabolic phenotypes. Specifically, when compliance with ORAC was high, minor-allele-carriers exhibited obesity-related traits such as BMI and $\mathrm{FM} \%$. On the other hand, improving the adherence to dietary FRAP might reduce the genetic association with insulin resistance indices (HOMA-IR and QUICKI) only among $G$ allele carriers (AG and GG). However, positive interactions of CARTPT rs2239670 on serum glucose level were found even in high compliance with FRAP and TEAC. Thus, the results of modification effect of diet on the associations of CARTPT with obesity and metabolic factors were not homogenous. According to our results, frequency of the rare allele $(20.79 \%)$ was nearly similar to the range of reported values in other population or ethnic groups such as Korean (17\%) [20] and Malaysian (30\%) [19]. This discrepancy in the minor allele frequency reported might be explained by differences in study sample size, demographic characteristics of population like age, ethnicity, and gender and also various lifestyles. Previous evidence has revealed that polymorphisms in the CARTPT gene are linked to human obesity. In this regard, leu34Phe missense mutation in CART gene was detected in Italian subjects with early-onset obesity [16]. Likewise, it was reported that the A$156 \mathrm{G}$ polymorphism in the promoter region of CARTPT was related to obesity among Japanese subjects [35]. Moreover, the studies have shown that genetic variation in the CART locus might influence susceptibility to MetS and its components such as dyslipidemia, high blood pressure and hyperglycemia [18]. It should be taken into account that all variants in CARTPT gene are not associated with obesity phenotypes and finding in this regard is conflicting [36]. For example, in accordance with the results of Walder study, C1442G variant of CARTPT gene was not associated with obesity in Pima Indians [36]. The previous studies which have specifically examined the association between the CARTPT rs2239670 polymorphism and addictive behaviors such as alcohol dependence have reported a positive association [20]. However, the studies which have evaluated this variant in relation to obesity are scarce. To the best of our knowledge, only one study have investigated the CARTPT rs2239670-obesity relation and showed no association between this variant and obesity among the Malaysian subjects [19]. All of these heterogeneities in finding warrants further research efforts among different populations. Since the rs2239670 variant located in the intron 1 of CARTPT gene and the strong effects on obesity and related 
metabolic factors may be removed during mRNA splicing encoding CART proteins, effects of this variant on CART function is still unknown [19]. However, genetic variation in the CART locus may effect on the expression of the CART peptide, which is related to hypothalamic anorectic and orexigenic neuropeptides [37]. Totally, it seems that CART peptides have an anorexigenic effect, although the mechanism of this function is not completely known [38].

As far as we are aware, no previous research has investigated the gene-diet interactions of CARTPT with adherence to the dietary NEAC on metabolic profile in obesity to compare our finding. However, there have been numerous researches investigating the interaction between genetic variation and diet or dietary ingredients on obesity and its-related complications [39]. For example, Mirzababaei and et al. examined the interaction of the rs1333048 variant on 9p21 genetic region with TAC on the risk of MetS and they revealed that high ORAC intake may modify the increased risk of MetS in homozygous subjects for the minor allele (AA genotype) [39]. Accordingly, in other our recent study, we documented the significant interactions between dietary quality indices and CARTPT rs2239670 genotypes affecting metabolic parameters [40]; higher adherence to diet quality index-international (DQI-I) reduced the metabolic risk in obese individuals with the homozygous minor allele genotype (AA).

Noticeably, the main finding of our study was the association between the CARTPT rs2239670 polymorphism and cardio-metabolic factors depended on the dietary antioxidant intakes; a good compliance with NEAC blunted the association of the CARTPT gene with metabolic factors. While the underlying mechanisms behind this interactions is not still clarified, these favorable effects of the total antioxidant capacity may be mediated by vitamin C, vitamin E and its isomers, selenium, carotenoids, isoflavones, flavonoids, and proanthocyanidins [41, 42]. In this regard, there are numerous studies which have indicated the beneficial effects of antioxidant-rich foods (e.g. fruits and vegetables and tea) on the obesity, insulin resistance, glucose homeostasis and lipid profiles $[43,44]$. These favorable effects of these antioxidants on the improvement of metabolic profile may be partly attributed to other activities of antioxidants such as regulation of brown adipose tissue metabolism and enhancement of thermogenesis, suppression of adipogenesis and induction of catabolism in adipose tissue [45].

The strengths of the present study is that according to our current knowledge, it is the first study to examine the interaction between CARTPT rs2239670 genotypes and dietary NEAC on the metabolic factors in obese subjects and identifying these gene-diet interactions may provide the best personalized dietary advice for high-risk participants according to their genetic makeup to reduce the burden of obesity and its-related chronic diseases.

However, the present study has several potential limitations that should be considered. Firstly, since the present study is a cross-sectional study, ascertained causality cannot be argued but it helps to generate hypotheses that can be examined by prospective cohort or other studies. Secondly, a rather small sample size of our study may not have sufficient statistical power to detect the interaction effect. So, the results of our study should be interpreted with caution and require replication and confirmation in larger and different populations. Thirdly, the present study was limited to the assessment of only one variant from a 
single gene, while multiple genes are known to be involved in the pathogenesis of obesity and its-related consequences. Fourthly, because dietary intakes and other lifestyle factors in Tabriz may differ from those in other parts of the country with different cultures, our results may not necessarily be extrapolated to the general population. Fifthly, despite adjustment for several confounders in the analyses, residual confounding by other unmeasured factors was inevitable. Lastly, under-reporting of dietary intakes particularly in obese individuals is a source of measurements error which may cause underestimation of the true effect [46]. Thus, we excluded the extreme-energy reporters from analysis.

\section{Conclusion}

In conclusion, our finding for the first time showed a statistically significant gene-diet interaction of the CARTPT rs2239670 with adherence to dietary NEAC, as a novel measurement of antioxidant intake, in relation to obesity and related- metabolic phenotypes; high intake of NEAC by minor-allele-carriers attenuated genetic association with BMI and FM\%, however high compliance with these indices couldn't effect on genetic predisposition to blood sugar abnormalities. Further studies are warranted to confirm our findings, which may have important implications for public health.

\section{Abbreviations}

NEAC, Non-Enzymatic Antioxidant Capacity, CARTPT, Cocaine and Amphetamine-Regulated Transcript Prepropeptide, FFQ, food frequency questionnaire; ORAC, oxygen radical absorbance capacity, FRAP, ferric reducing antioxidant power; TRAP, total radical trapping antioxidant parameter; TEAC, Trolox equivalent antioxidant capacity; CVDs, cardiovascular diseases; ROS, reactive oxygen species; MetS, metabolic syndromes.

\section{Declarations}

\section{Ethical approval and consent to participate}

Each participant was completely informed about the study protocol and provided a written informed consent form before taking part in the study. The study protocol was approved and registered by the ethics committee of Tabriz University of Medical Sciences (Ethics number: IR.TBZMED.REC.1397.266).

\section{Consent to publish}

Not applicable.

\section{Availability of data and materials}

All of the data are available with reasonable request from the corresponding author 
The authors declare that there is no conflict of interest.

\section{Funding}

The current work has been financially supported by research undersecretary of Tabriz University of Medical Sciences (identifier: IR.TBZMED.REC.1397.266).

\section{Authors' contributions}

All authors have read and approved the manuscript; MK, was involved in data collection, writing the first draft of the manuscript. MAF designed and supervised the project, revised the manuscript and supervised the project. MN was involved in lab works and data collection.

\section{Acknowledgement}

We thank all of the study participants.

\section{References}

1. Bluher M. Obesity: global epidemiology and pathogenesis. Nat Rev Endocrinol. 2019;15(5):288-98.

2. Chooi YC, Ding C, Magkos F. The epidemiology of obesity. Metabolism. 2019;92:6-10.

3. Bondia-Pons I, Ryan L, Martinez JA. Oxidative stress and inflammation interactions in human obesity. J Physiol Biochem. 2012;68(4):701-11.

4. Sosa V, Moline T, Somoza R, Paciucci R, Kondoh H, L.L. ME, Oxidative stress and cancer: an overview. Ageing Res Rev, 2013. 12(1): 376 - 90.

5. Csanyi G,FJ, Miller, Jr. Oxidative stress in cardiovascular disease. Int J Mol Sci. 2014;15(4):6002-8.

6. Herrera BM,CM, Lindgren. The genetics of obesity. Curr Diab Rep. 2010;10(6):498-505.

7. Frei B, Efficacy of dietary antioxidants to prevent oxidative damage and inhibit chronic disease. $J$ Nutr, 2004. 134(11): 3196s-3198 s.

8. Vieira AR, Abar L, Vingeliene S, Chan DS, Aune D, Navarro-Rosenblatt D, et al. Fruits, vegetables and lung cancer risk: a systematic review and meta-analysis. Ann Oncol. 2016;27(1):81-96.

9. Spence JD. Nutrition and stroke prevention. Stroke. 2006;37(9):2430-5.

10. Serafini M,D, Del, Rio. Understanding the association between dietary antioxidants, redox status and disease: is the Total Antioxidant Capacity the right tool? Redox Rep. 2004;9(3):145-52.

11. Pellegrini N, Serafini M, Colombi B, Del Rio D, Salvatore S, Bianchi M, et al. Total antioxidant capacity of plant foods, beverages and oils consumed in Italy assessed by three different in vitro assays. $J$ Nutr. 2003;133(9):2812-9. 
12. Colarusso L, Serafini M, Lagerros YT, Nyren O, La Vecchia C, Rossi M, et al. Dietary antioxidant capacity and risk for stroke in a prospective cohort study of Swedish men and women. Nutrition. 2017;33:234-9.

13. Parohan M, Sadeghi A, Khatibi SR, Nasiri M, Milajerdi A, Khodadost M, et al. Dietary total antioxidant capacity and risk of cancer: a systematic review and meta-analysis on observational studies. Crit Rev Oncol Hematol. 2019;138:70-86.

14. Mancini FR, Affret A, Dow C, Balkau B, Bonnet F, Boutron-Ruault MC, et al. Dietary antioxidant capacity and risk of type 2 diabetes in the large prospective E3N-EPIC cohort. Diabetologia. 2018;61(2):308-16.

15. Yu Z, Han S, Cao X, Zhu C, Wang X, Guo X. Genetic polymorphisms in adipokine genes and the risk of obesity: a systematic review and meta-analysis. Obesity (Silver Spring). 2012;20(2):396-406.

16. del Giudice EM, Santoro N, Cirillo G, D'Urso L, Di Toro R, Perrone L. Mutational screening of the CART gene in obese children: identifying a mutation (Leu34Phe) associated with reduced resting energy expenditure and cosegregating with obesity phenotype in a large family. Diabetes. 2001;50(9):215760.

17. Murphy KG. Dissecting the role of cocaine- and amphetamine-regulated transcript (CART) in the control of appetite. Brief Funct Genomic Proteomic. 2005;4(2):95-111.

18. Vasseur F, Guerardel A, Barat-Houari M, Cottel D, Amouyel P, Froguel P, et al. Impact of a CART promoter genetic variation on plasma lipid profile in a general population. Mol Genet Metab. 2007;90(2):199-204.

19. Lisa Y, Sook HF, Yee HS. Association of the Cocaine- and Amphetamine-Regulated Transcript Prepropeptide Gene (CARTPT) rs2239670 Variant with Obesity among Kampar Health Clinic Patrons, Malaysia. Malays J Med Sci. 2012;19(1):43-51.

20. Jung SK, Hong MS, Suh GJ, Jin SY, Lee HJ, Kim BS, et al. Association between polymorphism in intron 1 of cocaine- and amphetamine-regulated transcript gene with alcoholism, but not with bipolar disorder and schizophrenia in Korean population. Neurosci Lett. 2004;365(1):54-7.

21. Khodarahmi M, Asghari-Jafarabadi M, Abbasalizad Farhangi M. A structural equation modeling approach for the association of a healthy eating index with metabolic syndrome and cardiometabolic risk factors among obese individuals. PLoS One. 2019;14(7):e0219193.

22. Booth M. Assessment of physical activity: an international perspective. Res Q Exerc Sport. 2000;71(2 Suppl):114-20.

23. Matthews DR, Hosker JP, Rudenski AS, Naylor BA, Treacher DF, Turner RC. Homeostasis model assessment: insulin resistance and beta-cell function from fasting plasma glucose and insulin concentrations in man. Diabetologia. 1985;28(7):412-9.

24. Katz A, Nambi SS, Mather K, Baron AD, Follmann DA, Sullivan G, et al. Quantitative insulin sensitivity check index: a simple, accurate method for assessing insulin sensitivity in humans. J Clin Endocrinol Metab. 2000;85(7):2402-10. 
25. Mirmiran P, Esfahani FH, Mehrabi Y, Hedayati M, Azizi F. Reliability and relative validity of an FFQ for nutrients in the Tehran lipid and glucose study. Public Health Nutr. 2010;13(5):654-62.

26. Esfahani FH, Asghari G, Mirmiran P, Azizi F. Reproducibility and relative validity of food group intake in a food frequency questionnaire developed for the Tehran Lipid and Glucose Study. J Epidemiol. 2010;20(2):150-8.

27. Ghafarpour M, Houshiar-Rad A, Kianfar H, Ghaffarpour M. The manual for household measures, cooking yields factors and edible portion of food. 1999.

28. Khodarahmi M, Kahroba H, Jafarabadi MA, Mesgari-Abbasi M, Farhangi MA. Dietary quality indices modifies the effects of melanocortin-4 receptor (MC4R) rs17782313 polymorphism on cardiometabolic risk factors and hypothalamic hormones in obese adults. BMC Cardiovasc Disord. 2020;20(1):57-7.

29. Asghari G, Yuzbashian E, Mirmiran P, Azizi F. The association between Dietary Approaches to Stop Hypertension and incidence of chronic kidney disease in adults: the Tehran Lipid and Glucose Study. Nephrol Dial Transplant. 2017;32(suppl_2):ii224-30.

30. Carlsen MH, Halvorsen BL, Holte K, Bohn SK, Dragland S, Sampson L, et al. The total antioxidant content of more than 3100 foods, beverages, spices, herbs and supplements used worldwide. Nutr J. 2010;9:3.

31. Pellegrini N, Serafini M, Salvatore S, Del Rio D, Bianchi M, Brighenti F. Total antioxidant capacity of spices, dried fruits, nuts, pulses, cereals and sweets consumed in Italy assessed by three different in vitro assays. Mol Nutr Food Res. 2006;50(11):1030-8.

32. Wu X, Beecher GR, Holden JM, Haytowitz DB, Gebhardt SE, Prior RL. Lipophilic and hydrophilic antioxidant capacities of common foods in the United States. J Agric Food Chem. 2004;52(12):4026-37.

33. Delgado-Andrade C,FJ, Morales. Unraveling the contribution of melanoidins to the antioxidant activity of coffee brews. J Agric Food Chem. 2005;53(5):1403-7.

34. Morales FJ, Somoza V, Fogliano V. Physiological relevance of dietary melanoidins. Amino Acids. 2012;42(4):1097-109.

35. Yamada K, Yuan X, Otabe S, Koyanagi A, Koyama W, Makita Z. Sequencing of the putative promoter region of the cocaine- and amphetamine-regulated-transcript gene and identification of polymorphic sites associated with obesity. Int J Obes Relat Metab Disord. 2002;26(1):132-6.

36. Walder K, Morris C, Ravussin E. A polymorphism in the gene encoding CART is not associated with obesity in Pima Indians. Int J Obes Relat Metab Disord. 2000;24(4):520-1.

37. Hunter RG, Philpot K, Vicentic A, Dominguez G, Hubert GW, Kuhar MJ. CART in feeding and obesity. Trends Endocrinol Metab. 2004;15(9):454-9.

38. Ahmadian-Moghadam H, Sadat-Shirazi MS, Zarrindast MR. Cocaine- and amphetamine-regulated transcript (CART): A multifaceted neuropeptide. Peptides. 2018;110:56-77.

39. Mirzababaei A, Mollahosseini M, Rahimi MH, Yekaninejad MS, Maghbooli Z, Sobhani R, et al. Interaction between a variant of chromosome 9p21. 3 locus and diet antioxidant capacity on 
metabolic syndrome in Tehrani adults. Diabetol Metab Syndr. 2018;10(1):76.

40. Mahmoudi-Nezhad M, Farhangi MA, Kahroba H. Cocaine and amphetamine-regulated transcript prepropeptide gene (CARTPT) polymorphism interacts with Diet Quality Index-International (DQI-I) and Healthy Eating Index (HEI) to affect hypothalamic hormones and cardio-metabolic risk factors among obese individuals. J Transl Med. 2020;18(1):16.

41. Dakhale GN, Chaudhari HV, Shrivastava M. Supplementation of vitamin C reduces blood glucose and improves glycosylated hemoglobin in type 2 diabetes mellitus: a randomized, double-blind study. Adv Pharmacol Sci. 2011;2011:195271.

42. Manning PJ, Sutherland WH, Walker RJ, Williams SM, De Jong SA, Ryalls AR, et al. Effect of highdose vitamin $\mathrm{E}$ on insulin resistance and associated parameters in overweight subjects. Diabetes Care. 2004;27(9):2166-71.

43. Li M, Fan Y, Zhang X, Hou W, Tang Z. Fruit and vegetable intake and risk of type 2 diabetes mellitus: meta-analysis of prospective cohort studies. BMJ Open. 2014;4(11):e005497.

44. Maritim AC, Sanders RA, Watkins JB 3. Diabetes, oxidative stress, and antioxidants: a review. J Biochem Mol Toxicol. 2003;17(1):24-38. rd. . ).

45. Lee MS, Kim CT, Kim Y. Green tea (-)-epigallocatechin-3-gallate reduces body weight with regulation of multiple genes expression in adipose tissue of diet-induced obese mice. Ann Nutr Metab. 2009;54(2):151-7.

46. Fisher JO, Johnson RK, Lindquist C, Birch LL, Goran MI. Influence of body composition on the accuracy of reported energy intake in children. Obes Res. 2000;8(8):597-603.

\section{Figures}




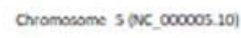

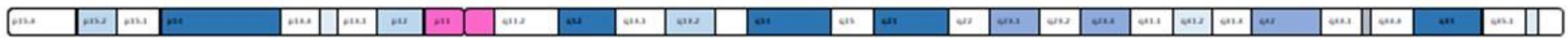

\begin{tabular}{|c|c|c|}
\hline ph, & 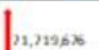 & 21,2n, \\
\hline
\end{tabular}

ona -40

\section{Figure 1}

Genomic organization of the CARTPT rs2239670 polymorphism region 

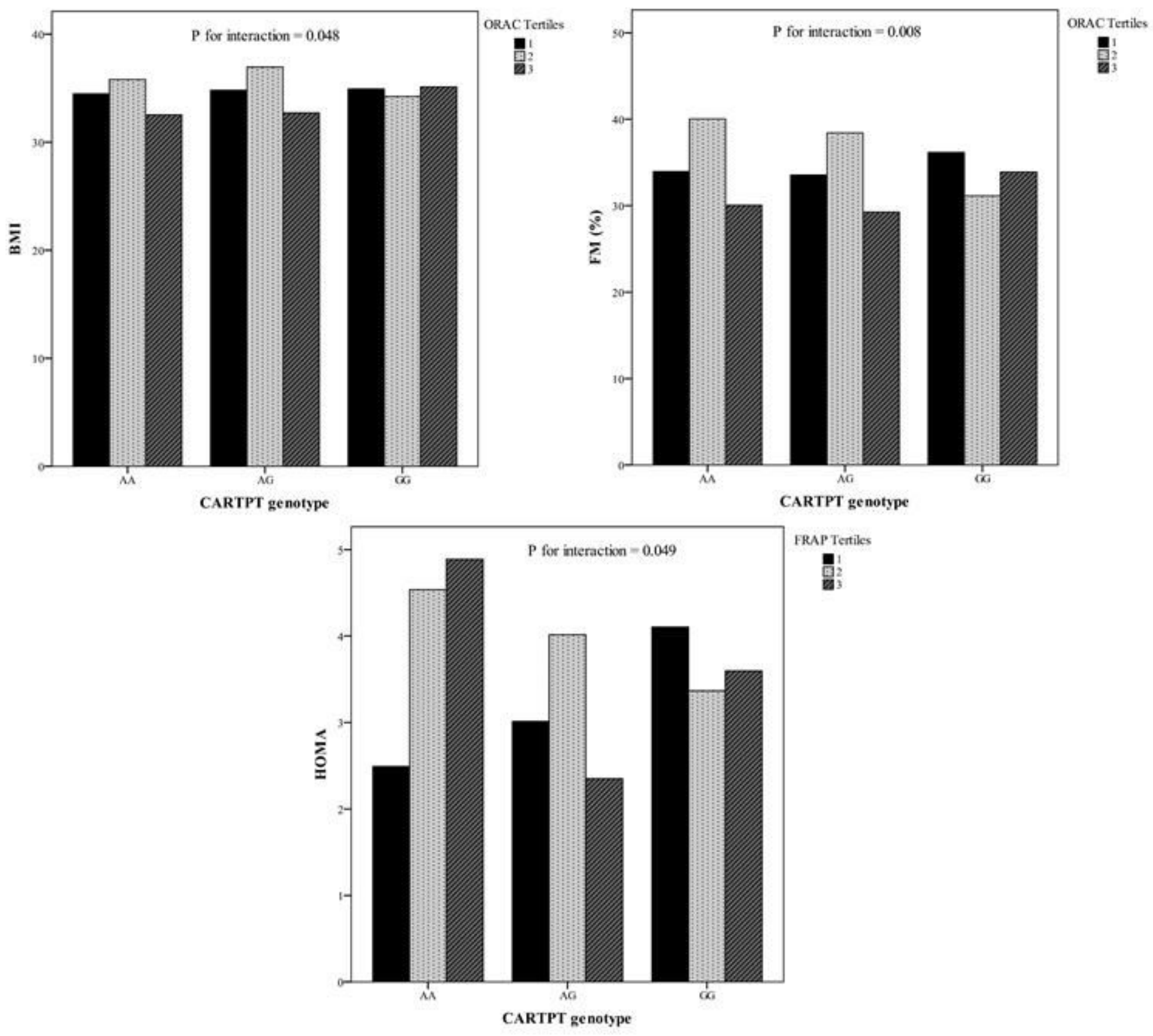

Figure 2

P-for interaction between different NEAC tertiles according to CARTPT genotype. 